\title{
Right Bundle Branch Block and Impaired Left Ventricular Function as Evidence of a Left Ventricular Conduction Delay
}

\author{
Hiroto Takamatsu, MD; Hiroshi Tada, MD; Hiroki Okaniwa, RDMS; Hiroyuki Toide, RDMS; \\ Hiroko Maruyama, RDMS; Rumi Higuchi, RDMS; Kenichi Kaseno, MD; Shigeto Naito, MD; \\ Masahiko Kurabayashi, MD*; Shigeru Oshima, MD; Koichi Taniguchi, MD
}

\begin{abstract}
Background The indications and efficacy of cardiac resynchronization therapy (CRT) have not been sufficiently clarified in patients with right bundle branch block (RBBB).

Methods and Results This study included 55 patients with normal QRS morphology and duration (ControlGr) and 49 patients with complete RBBB (CRBBB-Gr). Using tissue Doppler imaging, the time difference (TD) between the electromechanical delay of the septal wall, left ventricular (LV) lateral wall, and right ventricular free wall were measured. Using tissue tracking imaging, the coefficient of the time variation from the beginning of the QRS to the peak displacement time of 6 regions of the LV (CV-PMDLV) was calculated. The TD between the septal wall and that of the LV lateral wall (TDSEPT-LAT) did not differ between the Control-Gr and RBBB-Gr. However, a significant difference was found in the TDsEPT-LAT between the CRBBB patients with LV systolic dysfunction (ejection fraction $(E F) \leq 50 \%$ ) and those with normal LV function $(E F>50 \%$; $p<0.001$ ). The CVPMDLV was greater in the CRBBB patients with LV systolic dysfunction than in those with a normal LV function $(\mathrm{p}<0.05)$. The RBBB-Gr patients with LV dysfunction and a great TDSEPT-LAT, improved clinically after the CRT. Conclusions The presence of RBBB and LV dysfunction may indicate LV dyssynchrony and a heterogeneous mechanical dysfunction. (Circ J 2008; 72: 120-126)
\end{abstract}

Key Words: Heart failure; Right bundle branch block; Tissue Doppler imaging; Tissue tracking imaging; Ventricular dyssynchrony

C ardiac resynchronization therapy (CRT) in patients with advanced heart failure (HF) and a ventricular conduction delay improves symptoms and functional capacity, induces reverse remodeling, and reduces morbidity, and probably mortality $!^{1-4}$ It is well known that although the vast majority of CRT recipients present with left bundle branch block (LBBB), up to $10 \%$ of the minority have nonspecific conduction delays or right bundle branch block (RBBB) 2,5 Recently, RBBB was proven to be an important predictor of mortality in patients with congestive HF, just as has LBBB? However, patients with RBBB and congestive HF have not been adequately evaluated because of an under-representation in the referenced studies. Therefore, which type of patients with RBBB has ventricular conduction delays, and thus are good candidates for CRT, has not been fully clarified.

(Received February 5, 2007; revised manuscript received August 23, 2007; accepted August 31, 2007)

Division of Cardiology, Gunma Prefectural Cardiovascular Center, *Gunma University Graduate School of Medicine, Department of Medicine and Biological Science, Maebashi, Japan

Mailing address: Hiroshi Tada, MD, Division of Cardiology, Clinical Electrophysiology Section, Cardiovascular Center, CVC Cardiovascular Medicine, 1500E. Medical Center Dr., SPC 5853, Ann Arbor, MI48109-5853, USA. E-mail: htada@umich.edu

All rights are reserved to the Japanese Circulation Society. For permissions, please e-mail: cj@j-circ.or.jp

\section{Methods}

\section{Study Population}

This study included 104 patients (34 women, 70 men; mean age, $59 \pm 15$ years) who underwent conventional echocardiography, including tissue Doppler imaging (TDI) and tissue tracking imaging (TTI) between January 2002 and December 2004. Of these, 49 consecutive patients had complete RBBB (CRBBB) with a duration of the QRS complex of $\geq 120 \mathrm{~ms}$ (RBBB-Gr). The remaining 55 had normal morphology of the QRS complex and narrow QRS $(<120 \mathrm{~ms}$; Control-Gr), and were randomly selected so that the data collected would be statistically matched to the RBBB-Gr patients in terms of age and gender (Table 1). In total, 33 patients had hypertension, 5 dilated cardiomyopathy, 5 mitral valvular diseases, 2 hypertrophic cardiomyopathy, 1 congenital heart disease, 3 ischemic heart disease, and the remaining 93 (89\%) had no structural heart disease. Echocardiography demonstrated that the mean left ventricular ejection fraction (LVEF) was $64.2 \pm 11.8 \%$ (range, 12-83), and left ventricular end-diastolic diameter (LVEDD) 46.5 $8.5 \mathrm{~mm}$ (range, 34-75). All patients were in sinus rhythm, and the mean QRS duration was $113.8 \pm 33.3 \mathrm{~ms}$ (range, 70-200). All patients in the Control-Gr had LVEF $>50 \%$ (range, 51-83).

\section{Echocardiographic Examination Including TDI and TTI}

The left ventricular (LV) dimensions were measured by M-mode echocardiography in the parasternal long-axis view. The LVEDD was measured at the onset of the QRS 
complex, and the LV end-systolic dimension (LVESD) was defined as the smallest LV cavity?,8 Pulsed-wave TDI and TTI were performed with a Vivid 7 echocardiography system (GE Vingmed Ultrasound, Horten, Norway) after completing conventional echocardiography?,8 The images were obtained using a $1.5-4.0 \mathrm{MHz}$ phased array transducer (M3S transducer; GE Vingmed Ultrasound) at a depth of $10-16 \mathrm{~cm}$ in the LV apical 4-chamber view. The image sector width was set as narrow as possible to allow a frame rate acquisition greater than 100 frames/s. At least 2 cine loops of raw TDI data, each containing 3 cardiac cycles, were stored online as digital scan-line data using the Vivid 7 scanner and were retrieved later and analyzed using the built-in analysis tools.

The electromechanical conduction delays were measured by TDI 7,8 The region of interest was positioned at the basal portion of the septum and lateral wall of the LV and the free wall of the right ventricle (RV), and the time from the onset of the QRS to the peak myocardial sustained systolic velocity was measured (Figs 1A,B). The peak myocardial sustained systolic velocity was defined as the maximum velocity during systole, excluding the isovolumic contraction?, 8 The time difference between the electromechanical delay of the septal wall and that of the LV lateral wall (TDSEPT-LAT) was defined as the intraventricular conduction delay in the LV. We also measured the time difference between the electromechanical delay of the LV lateral wall and that of the RV free wall (TDRV-LAT) and that between the RV free wall and septum (TDRV-SEPT).

TTI enabled visualization of the longitudinal motion in each myocardial segment 9,10 It displayed the integral of the tissue velocity during systole, which equaled the distance motion along the Doppler axis during systole (Figs 1C,D). To assess LV synchrony, a quantitative tissue displacementtime curve was used for the analysis. Using this curve, the interval from the QRS onset to the peak myocardial displacement (PMD) in each region could be obtained (Fig 1D). We determined the coefficient of the variation in the time of the PMD in 6 regions (base, mid-point between the basal and apical portions, and mid-point between the 2 points on the
Table 1 Clinical Characteristics and Measurement Variables in the Control-Gr and RBBB-Gr Patients

\begin{tabular}{lccl}
\hline \hline & $\begin{array}{c}\text { Control-Gr } \\
(n=55)\end{array}$ & $\begin{array}{c}\text { RBBB-Gr } \\
(n=49)\end{array}$ & p value \\
\hline Gender $(M / F)$ & $34 / 21$ & $36 / 13$ & 0.21 \\
Age (years) & $56.4 \pm 14.7$ & $62.1 \pm 14.6$ & 0.0502 \\
Structural heart disease (\%) & $8(14.5)$ & $8(16.3)$ & 0.98 \\
LVEDD $(\mathrm{mm})$ & $45.9 \pm 4.8$ & $47.9 \pm 9.6$ & 0.18 \\
LVESD $(\mathrm{mm})$ & $28.5 \pm 4.5$ & $33.1 \pm 10.8$ & $<0.01$ \\
LVEF $(\%)$ & $68.3 \pm 6.7$ & $59.5 \pm 14.3$ & $<0.0001$ \\
QRS duration (ms) & $82.7 \pm 6.8$ & $145.3 \pm 19.8$ & $<0.0001$ \\
Medication $(\%)$ & & & \\
ARBs/ACEI & $4(7)$ & $8(16)$ & 0.26 \\
Ca antagonists & $12(21)$ & $6(12)$ & 0.30 \\
$\beta$-blockers & $6(11)$ & $6(12)$ & 0.92 \\
Digitalis & $0(0)$ & $4(8)$ & 0.10 \\
Diuretics & $1(2)$ & $6(12)$ & 0.08 \\
Antiarrhythmic drugs & $4(7)$ & $7(14)$ & 0.40 \\
TD & & & \\
TDSEPT-LAT (ms) & $15.3 \pm 17.5$ & $19.5 \pm 25.0$ & 0.33 \\
TDRV-LAT (ms) & $32.2 \pm 26.6$ & $55.2 \pm 32.1$ & $<0.0005$ \\
TDRV-SEPT (ms) & $23.1 \pm 20.7$ & $51.3 \pm 33.9$ & $<0.0001$ \\
CV-PMDLV (\%) & $3.7 \pm 2.9$ & $7.2 \pm 5.9$ & $<0.0005$ \\
\hline
\end{tabular}

Values are the mean \pm standard deviation.

The left ventricular (LV) lateral wall (TDSEPT-LAT) is the intraventricular conduction delay in the $L V$. The time difference $(T D)$ between the electromechanical conduction delay (EMD) of the septum and that of the left ventricular (LV) lateral wall (TDSEPT-LAT), EMD of the LV lateral wall and that of the right ventricular $(R V)$ free wall (TDRV-LAT), and EMD of the RV free wall and that of the septum (TDRV-SEPT) were determined by tissue Doppler imaging. The coefficient of variation in the time of the peak myocardial displacement in 6 regions of the $L V(C V-P M D L V)$ was determined by tissue tracking imaging. Control-Gr, normal QRS morphology and duration; RBBB-Gr, right bundle branch block with a duration of the QRS complex of $\geq 120 \mathrm{~ms} ; E D(S) D$, enddiastolic (systolic) dimension; $E F$, ejection fraction; $A R B$, angiotensin II receptor blocker; ACEI, angiotensin-converting enzyme inhibitor; Ca, calcium.

septal and lateral walls) of the LV (CV-PMDLv; Fig 1D)?

We confirmed that the regional myocardial velocity curves obtained by TDI and the PMD curve by TTI were identical or nearly identical, and 3 beats were measured and averaged for each measurement. Two experienced observers

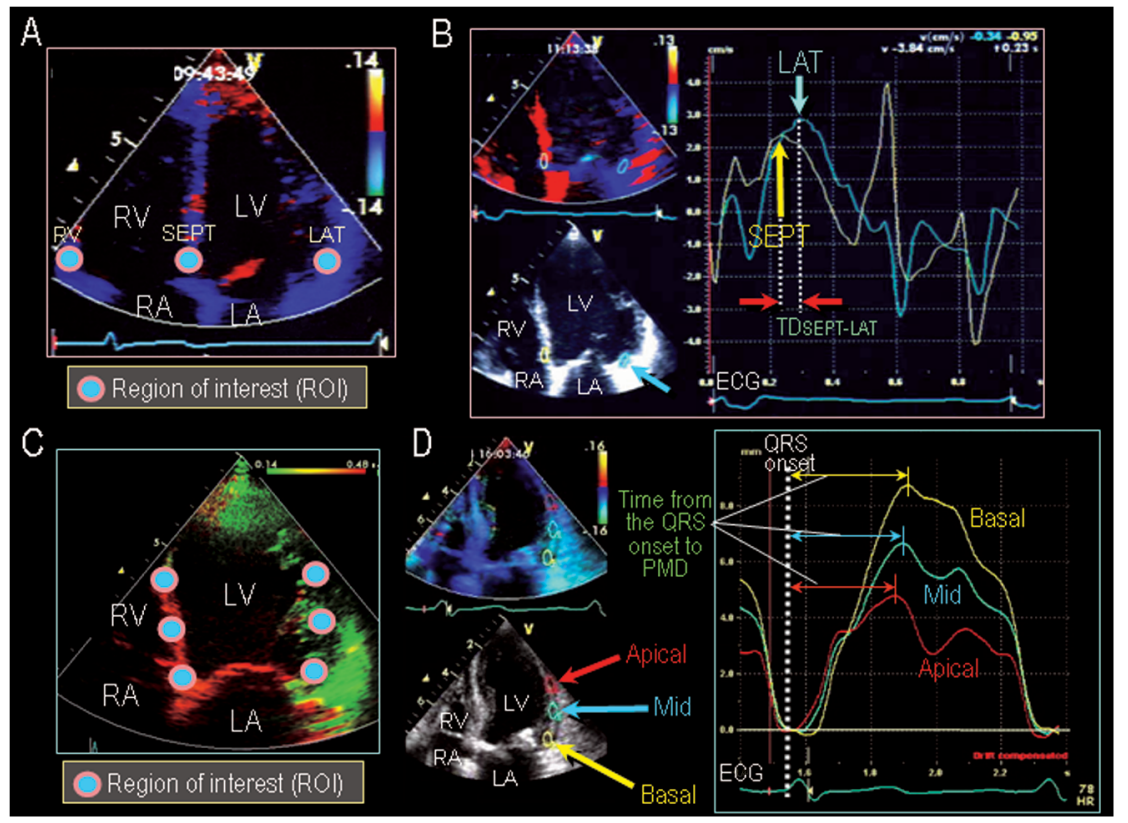

Fig 1. (A) Position of the region of interest (ROI) for the measurement of the time difference between the electromechanical delay of the septal wall (SEPT), left ventricular lateral wall (LAT), and that of the right ventricular free wall (RV). (B) Measurement of the time difference between the electromechanical delay of the septal wall and that of the left ventricle (LV) lateral wall (TDSEPTLAT). The ROI was positioned at the basal portion of the septum and lateral wall, and the time from the onset of the QRS to the peak myocardial sustained systolic velocity was measured. The time difference between these was defined as the TDsept-LAt. (C) Position of the ROIs for the measurement of the coefficient of variation in the time of the peak myocardial displacement (PMD) in 6 regions of the LV. (D) Quantitative tissue displacement-time curves obtained from the 3 regions of the LV lateral wall in a healthy control subject. The coefficient of variation in the time at which the PMD was recorded in 6 regions of the LV was calculated. $\mathrm{L}(\mathrm{R}) \mathrm{A}$, left (right) atrium. 
Table 2 Clinical Characteristics and Measurement Variables of the RBBB Patients With LV Systolic Dysfunction (LVEF $\leq \mathbf{5 0 \%}$ ) and Those With Normal LV Function (LVEF >50\%)

\begin{tabular}{|c|c|c|c|}
\hline & $\begin{array}{c}\text { Normal } L V \\
\text { function } \\
(n=41)\end{array}$ & $\begin{array}{l}\text { Reduced LV } \\
\text { function } \\
(n=8)\end{array}$ & $p$ value \\
\hline Gender $(M / F)$ & $30 / 11$ & $6 / 2$ & 0.74 \\
\hline Age (years) & $60.9 \pm 15.3$ & $68.3 \pm 8.3$ & 0.20 \\
\hline Structural heart disease (\%) & $1(2)$ & $7(88)$ & $<0.0001$ \\
\hline LVEDD $(\mathrm{mm})$ & $44.7 \pm 4.3$ & $64.4 \pm 12.5$ & $<0.0001$ \\
\hline $\operatorname{LVESD}(\mathrm{mm})$ & $29.0 \pm 4.0$ & $53.4 \pm 12.9$ & $<0.0001$ \\
\hline $\operatorname{LVEF}(\%)$ & $64.7 \pm 7.0$ & $32.8 \pm 12.3$ & $<0.0001$ \\
\hline QRS duration (ms) & $140.0 \pm 15.3$ & $172.5 \pm 18.3$ & $<0.01$ \\
\hline \multicolumn{4}{|l|}{ Medication (\%) } \\
\hline$A R B S / A C E I$ & $3(7)$ & $5(63)$ & $<0.001$ \\
\hline Ca antagonists & $5(12)$ & $1(13)$ & 0.57 \\
\hline$\beta$-blockers & $1(2)$ & $5(63)$ & $<0.0001$ \\
\hline Digitalis & $1(2)$ & $3(38)$ & $<0.01$ \\
\hline Diuretics & $0(0)$ & $6(75)$ & $<0.0001$ \\
\hline Antiarrhythmic drugs & $1(2)$ & $6(75)$ & $<0.0001$ \\
\hline \multicolumn{4}{|l|}{$T D$} \\
\hline TDSEPT-LAT $(\mathrm{ms})$ & $14.0 \pm 12.1$ & $47.4 \pm 48.9$ & $<0.0005$ \\
\hline TDRV-LAT ( $m s)$ & $53.1 \pm 31.4$ & $65.9 \pm 35.7$ & 0.31 \\
\hline TDRV-SEPT (ms) & $48.8 \pm 31.0$ & $64.0 \pm 46.8$ & 0.25 \\
\hline$C V-P M D L V(\%)$ & $6.4 \pm 5.1$ & $11.7 \pm 7.7$ & $<0.05$ \\
\hline
\end{tabular}

Values are the mean \pm standard deviation.

RBBB, right bundle branch block. Other abbreviations as in Table 1 .

who were familiar with TDI and TTI and unaware of the clinical status of the patients analyzed the data. In the RBBB$\mathrm{Gr}$, each parameter was also compared between patients with an impaired LV systolic dysfunction (LVEF $\leq 50 \%$ ) and those with normal LV function (LVEF > 50\%)!1,12

\section{Statistical Analysis}

All variables are expressed as the mean $\pm \mathrm{SD}$, and were compared using Student's paired t-test or non-parametric test as appropriate. The categorical variables were compared using a chi-squared analysis, using the Yate's correction if necessary. An overall Chi-square test for a $2 \times n$ table was constructed when comparisons involved $>2$ groups. Correlations between variables were assessed by Pearson's linear correlation and tested using Fisher's z transformation.
A p-value $<0.05$ was considered statistically significant.

\section{Results}

Clinical Characteristics, Measurement Variables, Electromechanical Conduction Delay, and CV-PMDLV in the Control-Gr and RBBB-Gr Patients

No significant difference was found between the 2 groups for gender, LVEDD, or frequency of using medications (Table 1). However, the patients in the RBBB-Gr had a greater LVESD $(\mathrm{p}<0.01)$ and lower LVEF $(\mathrm{p}<0.0001)$ than those in the Control-Gr (Table 1). No significant difference was found between the 2 groups for TDSEPT-LAT ( $\mathrm{p}=0.33$; Table 1), however, the TDRV-SEPT $(p<0.0001)$, TDRV-LAT $(\mathrm{p}<0.0005)$, and CV-PMDLV $(\mathrm{p}<0.0005)$ were greater in the RBBB-Gr patients than in the Control-Gr patients (Table 1). In the RBBB-Gr patients with normal LV function $(n=41$; LVEF >50\%; Table 2), the TDSEPT-LAT was comparable with that in the Control-Gr patients $(\mathrm{p}=0.68)$. However, the TDRV-SEPT $(\mathrm{p}<0.0001)$, TDRV-LAT $(\mathrm{p}<0.001)$, and CV-PMDLV $(p<0.005)$ were greater in the RBBB-Gr patients with normal LV function than in the Control-Gr patients.

Effect of LV Systolic Dysfunction on QRS Duration, Echocardiographic Measurements, and Electromechanical Conduction Delay in the RBBB-Gr Patients

Among the RBBB-Gr patients, the total duration of the QRS complex was greater in patients with LV systolic dysfunction $(\mathrm{n}=8)$ than in those with normal LV function (Table 2; $\mathrm{p}<0.01$ ). The LVEDD and LVESD were greater in the patients with LV systolic dysfunction than in those with normal LV function (both $\mathrm{p}<0.0001$; Table 2). There was no difference in the TDRV-SEPT $(\mathrm{p}=0.31)$ or TDRV-LAT $(\mathrm{p}=0.25)$ of the CRBBB patients with LV systolic dysfunction and that of the patients with a normal LV function (Table 2). However, there was a significant difference between the 2 groups for TDsEPT-LAT $(\mathrm{p}<0.001$; Table 2$)$. The CV-PMDLV was also greater in the CRBBB patients with LV systolic dysfunction than in those with normal LV function $(\mathrm{p}<0.05$; Table 2$)$.
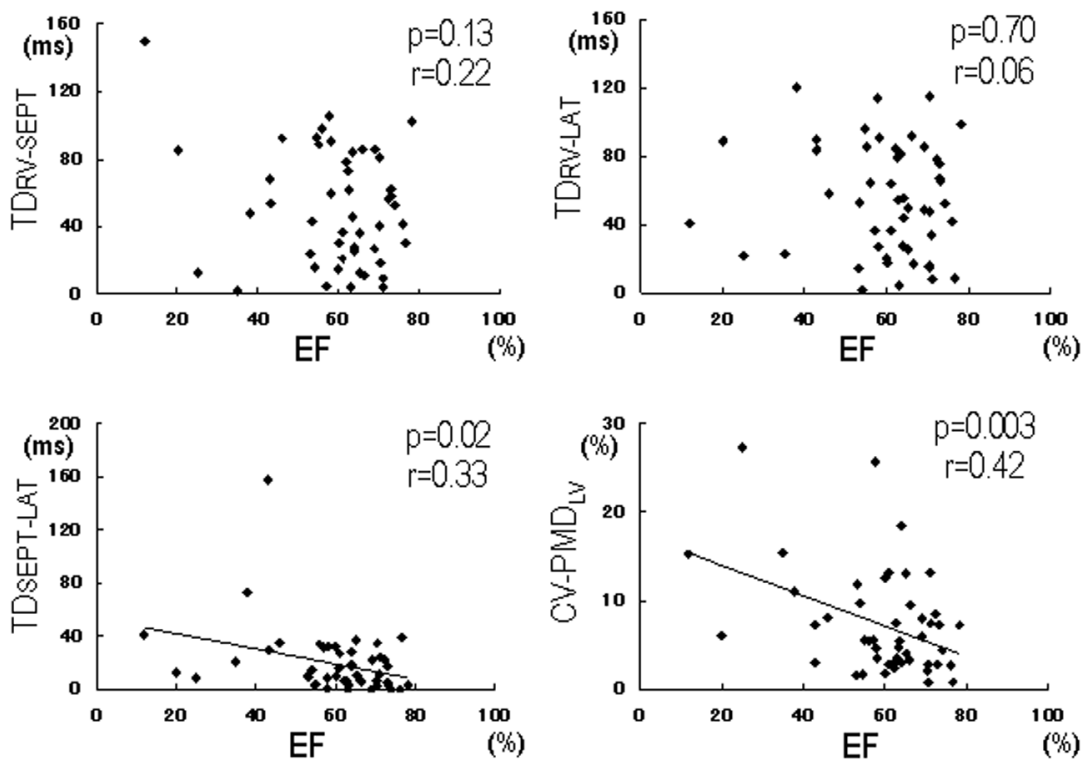

Fig 2. Correlation between the LVEF and the TDSEPT-LAT, TDRV-LAT, TDRV-SEPT, and CVPMDLv. See text for abbreviations. 

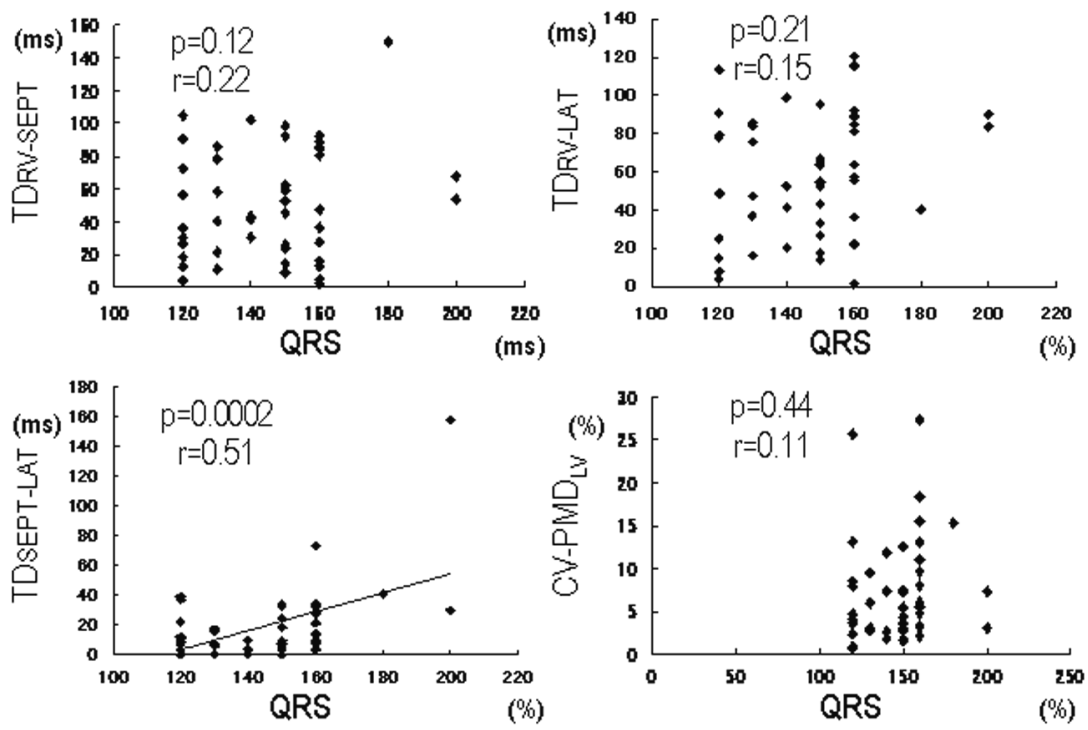

Fig 3. Correlation between the total QRS duration and the TDSEPT-LAT, TDRV-LAT, TDRVSEPT, and CV-PMDLV. See text for abbreviations.

Relationship of LVEF to the Conduction Delay and $C V$-PMDLV in the CRBBB Patients

No significant correlation was found between the LVEF and TDRV-SEPT $(\mathrm{p}=0.13, \mathrm{r}=0.22)$ or TDRV-LAT $(\mathrm{p}=0.70, \mathrm{r}=$ 0.06 ) in the CRBBB patients (Fig 2). However, a weak but significant correlation was found between the LVEF and TDSEPT-LAT $(\mathrm{p}=0.02, \mathrm{r}=0.33)$ and between the LVEF and CV-PMDLV ( $\mathrm{p}=0.003, \mathrm{r}=0.42 ;$ Fig 2$)$.

\section{Relationship of Total QRS Duration to the Conduction Delay and CV-PMDLV in the CRBBB Patients}

There was no significant correlation between the duration of the QRS complex and TDRV-SEPT $(\mathrm{p}=0.12, \mathrm{r}=0.22)$, TDRV-LAT $(\mathrm{p}=0.21, \mathrm{r}=0.15)$, or CV-PMDLV $(\mathrm{p}=0.44, \mathrm{r}=0.11)$ in any of the 49 CRBBB patients (Fig 3). However, a weak but significant positive correlation was found between the QRS duration and TDSEPT-LAT ( $\mathrm{p}=0.0002, \mathrm{r}=0.51$; Fig 3 ).

\section{Changes in the Measurement Variables Before and After} CRT in the CRBBB Patients

Five RBBB-Gr patients with LV dysfunction and advanced HF underwent CRT-P (Figs 4,5). The QRS duration was $>140 \mathrm{~ms}$ and the LVEF determined by echocardiography was $<35 \%$ in all of them. An atrial lead was implanted in the right atrial appendage, and the RV lead was placed at the RV apex? The LV pacing lead was inserted via the transvenous approach through the coronary sinus, and placed in the left marginal or posterolateral cardiac vein? One month after receiving CRT-P, 3 patients were classified as being responders who had clinically improved to New York Heart Association functional class of I or II and in whom the LV end-systolic volume decreased by $10 \%$. However, neither of the remaining 2 patients improved clinically nor did their LV end-systolic volume decrease by $10 \%$ (non-responders). There was no significant difference between the responders and non-responders for mean age $(\mathrm{p}=0.51)$, gender, $\mathrm{QRS}$ duration $(\mathrm{p}=0.27)$, LVEDD $(\mathrm{p}=0.70)$, LVESD $(\mathrm{p}=0.73)$ or LVEF $(\mathrm{p}=0.98)$ before receiving CRT-P.

No significant difference was found between the 2 groups for the TDSEPT-LAT ( $\mathrm{p}=0.18)$, TDRV-LAT $(\mathrm{p}=0.52)$, or TDRVSEPT $(\mathrm{p}=0.47)$ before receiving CRT-P. However, in the 3 responders, the TDSEPT-LAT before receiving CRT-P was greater than that in the 2 non-responders, and it decreased
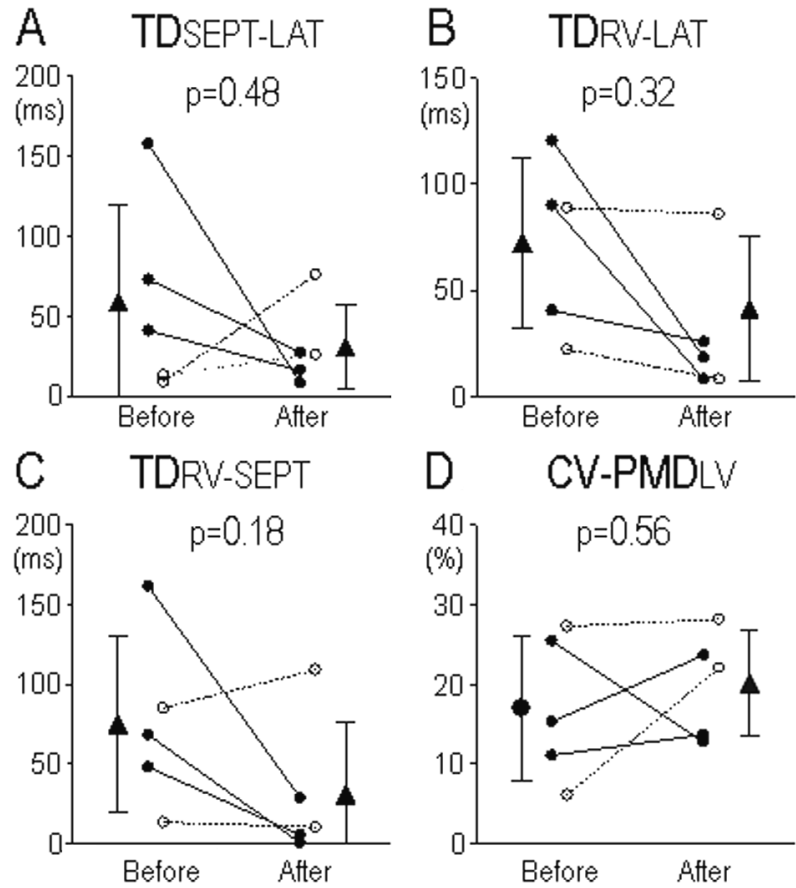

Fig 4. TDsept-Lat (A), TDrv-Lat (B), TDrv-Sept (C), and CVPMDLv (D) before and after CRT in responders (closed circles) and non-responders (open circles) among selected CRBBB patients with LV systolic dysfunction. See text for abbreviations.

after CRT-P (Fig 4). The TDRV-LAT and TDRV-SEPT before and after receiving CRT-P exhibited the same tendency (Fig 4). In the 2 non-responders, the TDSEPT-LAT before receiving CRT-P was quite small, comparable with that in the Control-Gr. One non-responder also had a small TDRV-LAT and TDRV-SEPT before receiving CRT-P. The other patient had a greater TDRV-LAT and TDRV-SEPT before receiving CRT-P, but neither decreased after CRT-P. The CV-PMDLV before receiving CRT-P varied in both groups $(\mathrm{p}=0.96)$, and did not improve in 4 of 5 patients after receiving CRT-P (Fig 4). 


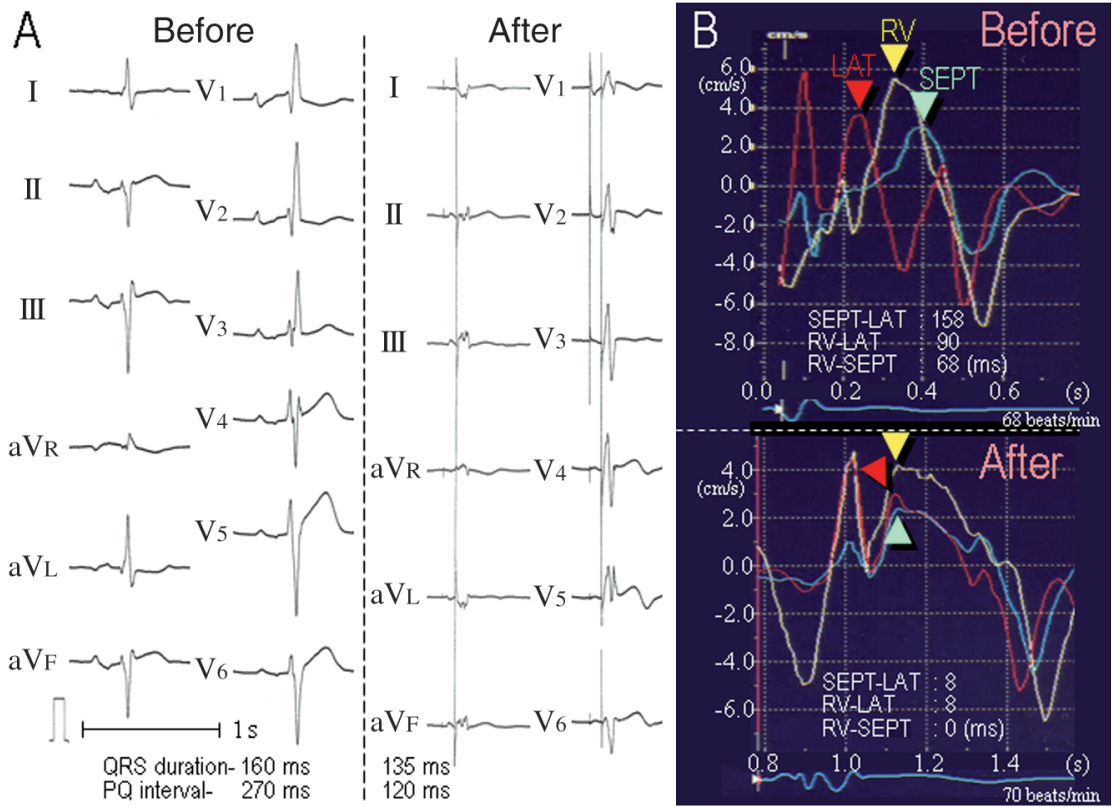

Fig 5. Representative case of CRBBB and LV systolic dysfunction before and after CRT (53-year-old female; in dilated phase of the non-obstructive hypertrophic cardiomyopathy; New York Heart Association (NYHA) functional class III). (A) 12-lead electrocardiograms. (B) TDI before and after CRT. Before CRT, there is a significant time difference between the electromechanical delay of the LV lateral wall (LAT) and septum (SEPT), the LAT and right ventricular free wall (RV), and the RV-SEPT, indicating that there is significant intra- and inter-ventricular dyssynchrony (Upper panel). However, after CRT, the LV dyssynchrony disappears (Lower panel). The severity of the mitral regurgitation became mild (grade II to grade I), and the LV ejection fraction improved from $43 \%$ to $65 \%$. The clinical status also improved to NYHA class I. See text for other abbreviations.

\section{Discussion}

\section{Major Findings}

From the measurements of ventricular dyssynchrony using TDI and TTI in patients with a normal QRS complex and CRBBB, the results of this study demonstrated the following findings.

(1) Although the TDRv-SEPT, TDrv-LAT, and CV-PMDlv were greater in the CRBBB-Gr patients than in the ControlGr patients, irrespective of the LVEF in the patients with CRBBB, the TDSEPT-LAT was comparable between the CRBBB patients and control subjects.

(2) In the CRBBB patients, no significant difference in the TDRV-SEPT or TDRV-LAT was found between the patients with a normal LV function and those with LV dysfunction. However, the TDSEPT-LAT, as well as the CV-PMDLv, was significantly greater in the patients with LV dysfunction, and there was a significant correlation between the LVEF and TDSEPT-LAT and between the LVEF and CV-PMDLV.

(3) The CRBBB patients with LV dysfunction and a high TDSEPT-LAT, improved clinically shortly after receiving CRT-P.

These results indicate that the presence of LV dysfunction (LVEF $<50 \%$ ) may indicate a great intra-LV dyssynchrony in CRBBB patients.

\section{QRS Width, CRBBB, LV Dyssynchrony, and CRT}

Several studies have demonstrated clinical improvement in CRT recipients with advanced HF and a wide QRS complex!-4 A widened QRS complex on the surface ECG is the most commonly used surrogate marker for interventricular dyssynchrony; ${ }^{13}$ however, it is becoming clear that the QRS duration may not always predict the clinical response, and that the prevalence of LV systolic asynchrony in patients with congestive HF and a normal QRS duration is not rare ${ }^{14-16}$ Therefore, the relationship between QRS duration and ventricular dyssynchrony is still controversial. A recent study indicated that the presence of an intra-LV (but not inter-V) asynchrony was an independent predictor of severe cardiac events, independent of LVEF and the QRS width! ${ }^{17}$ However, that study also demonstrated that the surface
ECG could identify patients with and without loco-regional contraction abnormalities only when the QRS duration was $>140 \mathrm{~ms}$, and that a QRS duration $>140 \mathrm{~ms}$ was found to be independently related to rehospitalization because of worsening of the HF ${ }^{17}$ In the present study, the CRBBB patients with LV systolic dysfunction had a longer QRS complex than those with normal LV function, and a significant positive correlation was found between QRS duration and TDSEPT-LAT. Therefore, QRS duration $>140 \mathrm{~ms}$ may be a marker for the presence of TDSEPT-LAT in CRBBB patients.

As with LBBB, CRBBB has been recently identified as an important and independent predictor of mortality in patients with congestive HF6 However, the majority of CRT recipients reported in previous studies have had LBBB QRS morphology,-3 and therefore the electrophysiological and mechanical rationale for using CRT in such patients has been well characterized ${ }^{18-20}$ In contrast there is little data for patients who present with nonspecific conduction delays or RBBB? 1 Thus, the relationship of CRBBB to ventricular dyssynchrony and the predictors of CRT effectiveness in CRBBB patients have not been fully clarified.

In the present study, the TDRV-SEPT and TDRV-LAT were significantly greater in the CRBBB patients than in the control subjects. In the patients with RBBB, a single RV breakthrough site appeared in the septum, and its activation slowly spread toward the anterior region and continued so that the latest activated regions were the right lateral wall and the outflow tract 21 The mechanism of the RV activation sequence in RBBB patients is considered to be most likely a result of cell-to-cell conduction, from the septum to the anterior wall, to the lateral wall, and finally to the outflow tract ${ }^{21}$ That mechanism may be responsible for the differences in the TDRV-SEPT and TDRV-LAT between the CRBBB patients and control subjects in the present study.

Among the CRBBB patients in the present study, a significant intraventricular conduction delay in the LV (TDRVSEPT) was observed in patients with LV systolic dysfunction, which was clearly different from the patients with normal LV systolic function. In the CRBBB patients, the CV-PMDLv, which is considered to reflect LV synchrony, was also significantly greater in the patients with LV sys- 
tolic dysfunction than in those with normal LV function. Schuster et al showed that even with a narrow QRS or RBBB, HF patients had a significant left-sided longitudinal asynchrony, as detected by color TDI ${ }^{17}$ Bader et al also reported that, in the patients with HF, the degree of intraventricular asynchrony, detected by TDI, was a better predictor of outcome than QRS duration or the $\mathrm{EF}$, and that no difference was found in the prevalence of such asynchrony in patients with RBBB or LBBB ${ }^{14}$ Furthermore, a recent study demonstrated that LV activation spreads slowly, from the septal or anterior breakthrough site, toward the apical and lateral regions, to the posterolateral basal region and around the mitral valve, which is the latest activated zone in HF patients with either RBBB or LBBB ${ }^{21}$ Thus, in CRBBB patients with advanced HF, LV activation is delayed as much as in the patients with isolated LBBB. In the present study, CRT was effective for the CRBBB patients with LV dyssynchrony. The previous studies and our results indicate that $L V$ conduction delay may be present in CRBBB patients with LV systolic dysfunction, and that CRT may be effective in those patients because more coordinated ventricular contractions are responsible for the beneficial effects of CRT. We believe that patients with RBBB and LBBB have a similar response to CRT when they have significant LV dyssynchrony.

\section{Role of TDI and TTI in Evaluating Ventricular Dysynchrony}

Among the noninvasive examination techniques, echocardiography, including TDI, is simple, easy and ideal for evaluating regional wall motion? $7,8,14,22,23 \mathrm{TDI}$ also enables accurate assessment of the regional timing of mechanical events relative to the phase of the cardiac cycle, and an intra- or inter-ventricular conduction delay can be easily measured by placing 2 sample volumes at the basal portion of the ventricle?.8 In the present study, using TTI, we also tested the utility of the CV-PMDLv as an index for evaluating LV dyssynchrony. Strain and strain rate imaging are useful for evaluating ventricular synchrony and cardiac performance?,22 However, these modalities are noise sensitive, especially in the setting of global LV dysfunction. TTI is not noise-sensitive, and has sufficient reproducibility and accuracy, with good spatial and temporal resolution and low inter- and intraobserver variabilities, ${ }^{9} 10$ In the present study, we could evaluate ventricular dyssynchrony using TDI and TTI, as well as the relationships between CRBBB and ventricular dyssynchrony and LV cardiac function. Our results were almost identical to those obtained from a study using an electro-anatomical mapping system ${ }^{21}$ Therefore, we think that measurement using TDI and TTI is a useful and reliable tool for evaluating the ventricular synchrony.

\section{Study Limitations}

First, this study had a relatively small sample size. In particular, the effect of CRT was examined in only 5 patients with CRBBB and LV dysfunction. The results obtained in those patients for TDI and TTI might indicate that a greater TDRV-LAT and TDRV-SEPT predict the effectiveness of CRT. Inversely, CV-PMDLv might be useless as a predictor for responders after receiving CRT. However, our sample size was too small to reach those conclusions. Therefore, future studies with a larger number of CRBBB patients receiving CRT are needed to clarify these points. Second, patients with both ischemic and non-ischemic CHF were included and therefore some patients with ischemic CHF would have had regional wall motion abnormalities that might affect mechanical dyssynchrony.

\section{Conclusions}

These results show that the presence of RBBB and LV dysfunction suggests LV dyssynchrony and heterogeneous mechanical dysfunction. A high intraventricular conduction delay in the LV may predict clinical improvement in patients with a CRBBB QRS morphology and LV dysfunction undergoing CRT.

\section{Acknowledgments}

We are indebted to Mr Yukihiro Yamamoto and Mr Tsutomu Nakajima, Ms Rika Utsugi, and the staff in the Cardiac Echo division for their important contribution to this study.

\section{References}

1. Abraham WT, Fisher WG, Smith AL, Delurgio DB, Leon AR, Loh E, et al. Multicenter InSync Randomized Clinical Evaluation: Cardiac resynchronization in chronic heart failure. N Engl J Med 2002; 346: $1845-1853$.

2. Bristow MR, Saxon LA, Boehmer J, Krueger S, Kass DA, De Marco T, et al. Comparison of Medical Therapy, Pacing, and Defibrillation in Heart Failure (COMPANION): Cardiac-resynchronization therapy with or without an implantable defibrillator in advanced chronic heart failure. N Engl J Med 2004; 350: 2140-2150.

3. Cleland JG, Daubert JC, Erdmann E, Freemantle N, Gras D, Kappenberger L, et al. Cardiac Resynchronization-Heart Failure (CARE-HF) Study Investigators: The effect of cardiac resynchronization on morbidity and mortality in heart failure. $N$ Engl J Med 2005; 352: 1539-1549.

4. Higuchi K, Toyama T, Tada H, Naito S, Ohshima S, Kurabayashi M. Usefulness of biventricular pacing to improve cardiac symptoms, exercise capacity and sympathetic nerve activity in patients with moderate to severe chronic heart failure. Circ J 2006; 70: 703-709.

5. Moss AJ, Zareba W, Hall WJ, Klein H, Wilber DJ, Cannom DS, et al; Multicenter Automatic Defibrillator Implantation Trial II Investigators. Prophylactic implantation of a defibrillator in patients with myocardial infarction and reduced ejection fraction. $N$ Engl J Med 2002; 346: $877-883$.

6. Hesse B, Diaz LA, Snader CE, Blackstone EH, Lauer MS. Complete bundle branch block as an independent predictor of all-cause mortality: Report of 7,073 patients referred for nuclear exercise testing. Am J Med 2001; 110: 318-319.

7. Tada H, Toide H, Naito S, Kurosaki K, Ito S, Miyaji K, et al. Tissue Doppler imaging and strain Doppler imaging as modalities for predicting clinical improvement in patients receiving biventricular pacing. Circ J 2005; 69: 194-200.

8. Tada H, Toide H, Okaniwa H, Higuchi R, Nakajima T, Utsugi R, et al. Maximum ventricular dyssynchrony predicts the clinical improvement and reverse remodeling in patients receiving biventricular pacing. Pacing Clin Electrophysiol 2007; 30: S13-S18.

9. Pan C, Hoffmann R, Kuhl H, Severin E, Franke A, Hanrath P. Tissue tracking allows rapid and accurate visual evaluation of left ventricular function. Eur J Echocardiogr 2001; 2: 197-202.

10. Sun JP, Chinchoy E, Donal E, Popovic ZB, Perlic G, Asher CR, et al. Evaluation of ventricular synchrony using novel Doppler echocardiographic indices in patients with heart failure receiving cardiac resynchronization therapy. J Am Soc Echocardiogr 2004; 17: 845850.

11. Wang TJ, Evans JC, Benjamin EJ, Levy D, LeRoy EC, Vasan RS. Natural history of asymptomatic left ventricular systolic dysfunction in the community. Circulation 2003; 108: 977-982.

12. Redfield MM, Jacobsen SJ, Burnett JC, Mahoney DW, Bailey KR, Rodeheffer RJ. Burden of systolic and diastolic ventricular dysfunction in the community: Appreciating the scope of the heart failure epidemic. JAMA 2003; 289: 194-202.

13. Auricchio A, Stellbrink C, Butter C, Sack S, Vogt J, Misier AR, et al; Pacing Therapies in Congestive Heart Failure II Study Group; Guidant Heart Failure Research Group. Clinical efficacy of cardiac resynchronization therapy using left ventricular pacing in heart failure patients stratified by severity of ventricular conduction delay. $J$ Am Coll Cardiol 2003; 42: 2109-2116.

14. Bader H, Garrigue S, Lafitte S, Reuter S, Jais P, Haissaguerre M, et 
al. Intra-left ventricular electromechanical asynchrony: A new independent predictor of severe cardiac events in heart failure patients. $J$ Am Coll Cardiol 2004; 43: 248-256

15. Achilli A, Sassara M, Ficili S, Pontillo D, Achilli P, Alessi C, et al. Long-term effectiveness of cardiac resynchronization therapy in patients with refractory heart failure and "narrow" QRS. J Am Coll Cardiol 2003; 42: 2117-2124.

16. Yu CM, Chau E, Sanderson JE, Fan K, Tang MO, Fung WH, et al. Tissue Doppler echocardiographic evidence of reverse remodeling and improved synchronicity by simultaneously delaying regional contraction after biventricular pacing therapy in heart failure. Circulation 2002; 105: 438-445.

17. Schuster P, Faerestrand S, Ohm OJ. Color Doppler tissue velocity imaging can disclose systolic left ventricular asynchrony independent of the QRS morphology in patients with severe heart failure. Pacing Clin Electrophysiol 2004; 27: 460-467.

18. Auricchio A, Fantoni C, Regoli F, Carbucicchio C, Goette A, Geller $\mathrm{C}$, et al. Characterization of left ventricular activation in patients with heart failure and left bundle-branch block. Circulation 2004; 109: $1133-1139$
19. Rodriguez LM, Timmermans C, Nabar A, Beatty G, Wellens HJ. Variable patterns of septal activation in patients with left bundle branch block and heart failure. J Cardiovasc Electrophysiol 2003; 14: $135-141$.

20. Grines CL, Bashore TM, Boudoulas H, Olson S, Shafer P, Wooley CF. Functional abnormalities in isolated left bundle branch block: The effect of interventricular asynchrony. Circulation 1989; 79: $845-853$.

21. Fantoni C, Kawabata M, Massaro R, Regoli F, Raffa S, Arora V, et al. Right and left ventricular activation sequence in patients with heart failure and right bundle branch block: A detailed analysis using threedimensional non-fluoroscopic electroanatomic mapping system. $J$ Cardiovasc Electrophysiol 2005; 16: 112-119.

22. Bax JJ, Abraham T, Barold SS, Breithardt OA, Fung JW, Garrigue S, et al. Cardiac resynchronization therapy. Part 1: Issues before device implantation. J Am Coll Cardiol 2005; 46: 2153-2167.

23. Bax JJ, Ansalone G, Breithardt OA, Derumeaux G, Leclercq C, Schalij MJ, et al. Echocardiographic evaluation of cardiac resynchronization therapy: Ready for routine clinical use? A critical appraisal. J Am Coll Cardiol 2004; 44: 1-9. 